\title{
Metodologia Não Destrutiva de Determinação da Indentação no Processo de Soldagem a Ponto por Resistência Elétrica em Chapas de Aços Galvanizados
}

\author{
Adriano Cássio Baldim ${ }^{1}$, Sebastião Carlos da Costa $^{1}$, Carlos Andrés Arango Hincapié ${ }^{1}$, Roney Fonseca ${ }^{1}$, \\ Thairone Conti Serafini Aguiar ${ }^{1}$ \\ 1 Universidade Federal de Itajubá - UNIFEI, Instituto de Engenharia Mecânica, Laboratório de Soldagem, Itajubá, MG, Brasil.
}

Recebido: 02 Mar., 2017

Aceito: 21 Jun., 2017

E-mails: adriano baldim@yahoo.com.br (ACB), sccosta@unifei.edu.br (SCC), carlos.arangoh@gmail.com (CAAH), roneyfonseca123@terra.com.br (RF), thaironemg@hotmail.com (TCSA)
Este é um artigo publicado em acesso aberto (Open Access) sob a licença Creative Commons Attribution Non-Commercial, que permite uso, distribuição e reprodução em qualquer meio, sem restriçōes desde que sem fins comerciais e que 0 trabalho original seja corretamente citado.
Resumo: A soldagem das chapas de aço galvanizado, em sua grande maioria é realizada através do processo de soldagem a ponto por resistência elétrica, principalmente no setor automotivo. Apesar de suas vantagens, no momento em que ocorre a fusão a pressão exercida pelo eletrodo vence a resistência do material culminando em uma leve penetração do eletrodo no metal base. Este fenômeno é denominado de indentação. Neste contexto, este trabalho estudou a indentação do ponto soldado, com o objetivo de determinar a penetração do eletrodo por meio de um método não destrutivo. Para isto, foram avaliados os sinais gerados por um sensor que registra o deslocamento do eletrodo durante as fases do processo de soldagem a ponto, utilizando como base, a leitura da expansão térmica da chapa de aço galvanizado. A profundidade da indentação foi revelada graficamente pelo sensor de deslocamento do eletrodo em comparação a medição realizada com o relógio apalpador por métodos estatísticos. Evidências mostraram que o sinal de deslocamento do eletrodo, mostrou ser um eficiente método na determinação da indentação no processo de soldagem a ponto por resistência elétrica em chapas de aços galvanizados.

Palavras-chave: Indentação; Deslocamento do eletrodo; Soldagem a ponto; Aço galvanizado.

\section{Non Destructive Methodology for Determination of the Indentation in the Resistance Spot Welding Process in Steel Plates Galvanized}

\begin{abstract}
The welding of galvanized steel plates, mostly takes place via the welding process to the resistance spot welding, especially in the automotive sector. Despite its advantages, which occurs when the melt pressure exerted by the electrode overcomes the resistance of the material culminating in a light penetration of the electrode base metal. This phenomenon is called indentation. In this context, this paper studied the indentation spot welded, in order to determine the penetration of the electrode through a non-destructive method. To this, were evaluated signals generated by a sensor which registers the displacement of the electrode during the phases of the welding process to the point, using as basis, the reading of thermal expansion of the galvanized steel sheet. The deepness of indentation was revealed graphically by the electrode of the displacement sensor compared the measurement performed with the probe clock by statistical methods. Evidence has shown that the electrode displacement signal, proved to be an efficient method for determination of the indentation in the welding process to the point electric resistance of galvanized steel sheets.
\end{abstract}

Key-words: Indentation; Electrode displacement; Spot welding; Galvanized steel.

\section{Introdução}

Considerando os vários processos de soldagem existentes em um ambiente industrial, a soldagem a ponto por resistência elétrica (RSW do inglês Resistance Spot Welding) tem se destacado principalmente no setor automotivo, que atualmente é considerado o setor que mais emprega este processo. Fato este, é devido a sua alta produtividade, facilidade de operação e automação, possibilidades de união de chapas finas, não utilização de consumíveis e, rapidez de execução associado a um baixo custo. Marques et al. [1] mostra que o processo RSW também tem sido muito utilizado em outros setores industriais como eletrônico, naval e outras indústrias de bens manufaturados. Neste contexto, as investigações científicas ligadas aos processos de soldagem são de extrema importância para o avanço tecnológico na área de fabricação mecânica abrindo possibilidades para a obtenção de 
produtos de alta qualidade [2,3]. Muito embora, sua resistência à corrosão e baixo custo em comparação a outros metais que possuem a mesma propriedade justifique sua grande utilização no setor automotivo, a presença do zinco no revestimento além de ser um fator complicador, culmina na utilização de maiores valores de corrente e tempo de soldagem [4]. Para tanto, a garantia da qualidade da soldagem ganha destaque quando se refere à segurança dos passageiros e também à vida útil dos veículos [5,6]. A qualidade da soldagem RSW é influenciada por vários fatores que ocorrem durante o processo. Segundo Zhou et al. [7], o entendimento destes fatores e seus efeitos são importantes para se garantir a qualidade da solda a ponto. Tais fatores ditos controláveis podem ser a corrente de soldagem, a pressão do eletrodo e o tempo de soldagem. Por outro lado, os fatores incontroláveis, podem ser identificados como o encaixe das chapas, o desgaste no eletrodo e as impurezas presentes nos processos industriais como óleos, graxas e os óxidos, sempre aparentes nas superfícies das chapas. A combinação destes fatores influencia na geometria e por consequência na qualidade final do ponto soldado e desta forma, necessitam ser avaliados adequadamente seguindo para tanto especificações de normas vigentes. Sendo assim, a necessidade de monitorar a qualidade dos pontos de solda se torna mais evidente. Uma forma de avaliação utilizada hoje nas indústrias para verificar a qualidade final do ponto de solda são os ensaios destrutivos. Este método, além de ser feito por amostragem, não representa claramente a condição real do conjunto soldado. Desta forma, os fatores incontroláveis passam despercebidos.

Muitas pesquisas têm sido feitas na tentativa de monitoramento não destrutivo do ponto de solda [8]. Todavia, a presença dos fatores incontroláveis possa contribuir para o aumento da resistividade elétrica que dificulta a passagem de corrente entre os eletrodos, a geometria do ponto soldado pode ser garantida pelo aumento automático da amperagem. Este aumento é aplicado por meio de equipamentos eletrônicos que ao perceber a diminuição da passagem de corrente entre os eletrodos, aumenta automaticamente a corrente aplicada na soldagem [9]. Por outro lado, este acréscimo de corrente elétrica no equipamento de soldagem aumenta a energia de soldagem aplicada no metal, contribuindo para uma maior expansão térmica e o aumento da ductilidade do metal a ser soldado [10]. Quanto maior o aporte térmico, mais dúctil se torna o material e a força do eletrodo vence a resistência do aço. Assim, os eletrodos penetram no metal base [11] e quanto maior for o aquecimento, maior é a indentação (por penetração nas interfaces externas) causada pelo eletrodo na chapa. É desejável, que ela seja mínima possível dentro de um limite tolerável dependendo do setor industrial [7]. 0 limite máximo de aceitação da indentação observado pela maioria das indústrias automobilísticas corresponde a $20 \%$ da espessura das chapas soldadas [12]. Por outro lado, considerando que a AWS/SAE D8.9M [13] não indica nenhum limite máximo para a indentação, tem-se a liberdade de variação da mesma, mediantes os resultados de aparência e resistência exigidas pelos clientes [14]. Todavia, durante um ensaio de fadiga o excesso de indentação contribui para a diminuição da vida do componente soldado [15]. Segundo Xinmin et al. [16], o método de inspeção de qualidade de solda on-line que se baseia na indentação, pode distinguir com precisão as peças soldadas que estão fora dos requisitos de resistência, além de diagnosticar falhas de soldagem. Neste contexto, considerando que a indentação esteja relacionada com a energia de soldagem e que, a mesma tenha relação com a variação dos parâmetros de soldagem [5]; para este trabalho foi utilizado um sensor que irá captar a dilatação e a variação da expansão térmica do material a ser soldado ao longo do processo e, por meio de um transdutor estes dados são decodificados em dados numéricos e assim, por meio de uma interface gráfica, todo processo de soldagem é demonstrado, revelando o deslocamento do eletrodo durante o processo RSW. Alguns autores como Lee e Choo [11], observaram que o sinal de deslocamento do eletrodo está diretamente ligado à formação do ponto de solda. Gedeon e Eagar [17], disseram ser possível utilizar as curvas de deslocamento do eletrodo, para caracterizar fisicamente todo o processo de soldagem. Com base nas informações até o momento, este trabalho procurou validar por meios de estudos estatísticos [18,19] uma metodologia não destrutiva a partir de um sensor de deslocamento, para caracterizar o fenômeno da indentação durante a soldagem por resistência elétrica em chapas de aço galvanizado. Para tanto, mediante sua correlação com o relógio apalpador, foi utilizado o deslocamento do eletrodo como sinal efetivo de alta confiabilidade na descrição do comportamento deste fenômeno.

\section{Materiais e Métodos}

Este capítulo descreve todas as etapas do procedimento experimental realizadas tais como adaptação do banco de ensaios, conjugadas com a utilização de um sensor de deslocamento indutivo para avaliação do deslocamento dos eletrodos durante o período de preaquecimento e soldagem. Da mesma forma, estão os materiais e equipamentos utilizados, bem como os níveis dos parâmetros selecionados, para a determinar a influência 
da variação do sinal do sensor sobre a indentação medida por meio de um relógio apalpador, em soldagens de chapas de aços galvanizados. Todo o experimento foi realizado no Laboratório de Soldagem - LSO da Unifei, em

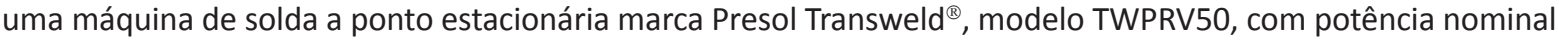
de 50 kVA, CA, alimentação de $220 \mathrm{~V}$ e corrente máxima de 6kA, cujos detalhes de seus principais acessórios são mostrados na Figura 1B. Acoplado ao equipamento de soldagem a ponto, foi utilizado um transdutor diferencial de deslocamento linear (LVDT), modelo GA-HD LBB315PA-100-M, fabricante Metrolog ${ }^{\circledR}$, com suporte do eixo por rolamento linear de precisão, avanço do êmbolo por mola e apalpador de carbono-tungstênio com repetibilidade de 0,0001mm e faixa de medição de +/- 2,5mm.

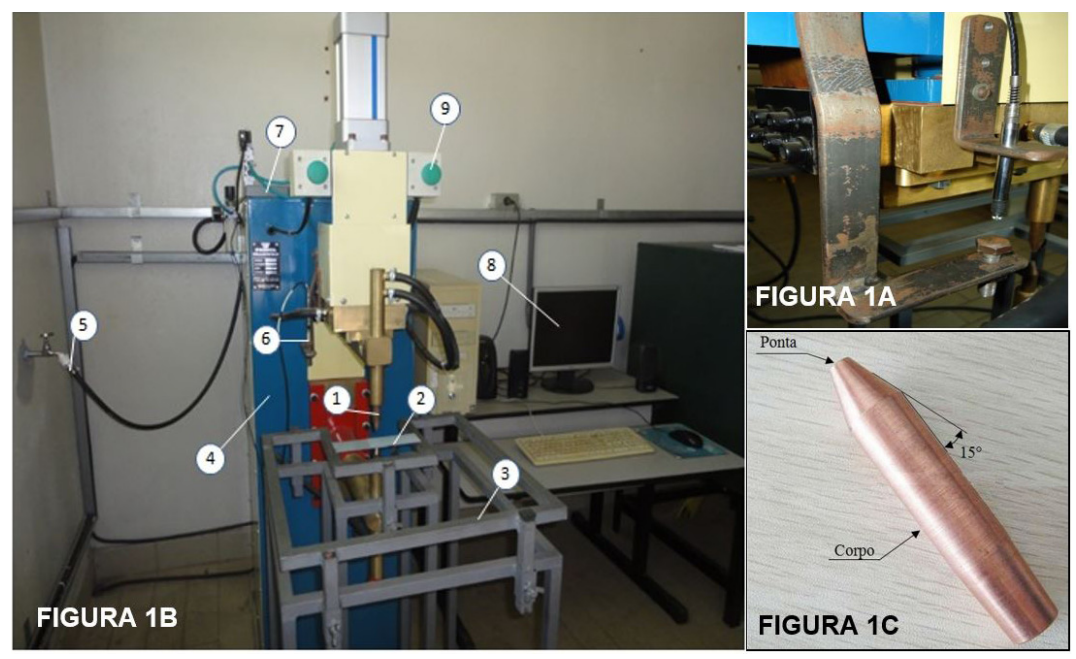

Figura 1. Equipamento estacionário de soldagem RSW e seus componentes: (A) Sensor LVDT Metrolog; (B) Sistema completo de soldagem - 1. Eletrodo, 2. Corpo de prova, 3. Dispositivo para fixar o corpo de prova, 4. Estacionária Presol TWPRV50 6kA, 5. Água de refrigeração, 6. Sensor, 7. Condicionador digital Metrolog SD $20,8$. Interface, 9. Botão de acionamento pneumático; (C) Eletrodo cônico de ponta truncada. Fonte: Baldim [8].

O sensor do sistema de medição foi adaptado ao cabeçote móvel da máquina de solda. Este por sua vez, está conectado ao cilindro pneumático que efetua o movimento do eletrodo superior. $\mathrm{O}$ ajuste da referência do sensor se dá por um parafuso sextavado de cabeça chata que está fixado em uma base parafusada na parte fixa da carcaça da máquina de solda conforme é mostrado na Figura $1 \mathrm{~A}$. O deslocamento linear do transdutor é medido por meio de uma interface, constituída por um sistema de aquisição dos dados formado por um condicionador digital Metrolog ${ }^{\circledR}$, modelo SD20 com saída USB para aplicação com sensores LVDT e conversor A/D 24-bits.

Para o desenvolvimento dos trabalhos foram utilizados eletrodos de cobre cromo zircônio, Grupo A, classe 2, com formato cone truncado a 15으, com diâmetro da ponta de $5 \mathrm{~mm}$, diâmetro do corpo 5/8" e comprimento total de $70 \mathrm{~mm}$, conforme Figura 1C. Para atingir um desempenho adequado, os eletrodos necessitam ser refrigerados com a intenção de evitar sua degradação, sendo este, um procedimento fundamental recomendado por fabricantes a ser checado antes da realização das soldagens. Neste caso específico, uma vazão d'água de $8 \mathrm{l} / \mathrm{min}$. foi ajustada. Após sua retificação, foram realizados de 30 - 50 pontos de solda em uma chapa de aço zincada com intuito de formação de uma fina camada de latão na ponta do eletrodo. Este procedimento, denominado "amaciamento" por Azambuja [4], evita que os eletrodos novos grudem a chapa zincada no início da soldagem. Por último, a Tabela 1 mostra, a composição química do corpo de prova de $1 \mathrm{~mm}$ de espessura da chapa utilizado no experimento.

Tabela 1. Composição química do aço galvanizado utilizado.

\begin{tabular}{ccccccc}
\hline Material & $\boldsymbol{C}$ & $\boldsymbol{M n}$ & $\boldsymbol{P}$ & $\boldsymbol{S}$ & $\boldsymbol{A l}$ & $\mathbf{Z n}$ \\
$\mathbf{\%}$ & $\%$ & $\%$ & $\mathbf{9} / \mathbf{m}^{\mathbf{2}}$ \\
Aço carbono USIGALVE-EEP-PC & 0,06 & 0,35 & 0,025 & 0,025 & 0,02 & $40-60$ \\
\hline
\end{tabular}


Em todos os testes, a pressão dos eletrodos foi considerada em $430 \mathrm{kPa}$. Após a soldagem, a pressão se manteve pelo tempo de 40 ciclos, para uma melhor formação do ponto de solda [20]. Esta fase culminou com a definição dos níveis dos parâmetros selecionados para a pesquisa em questão, considerando um projeto de experimentos estatísticos DOE. Foram realizados testes de simulação física, com intuito de determinar os limites iniciais e finais para cada parâmetro de tempo de preaquecimento (T.preaq), de corrente de preaquecimento (I.preaq), de tempo de soldagem (T.solda) e de corrente de soldagem (I.solda) conforme mostrados na Tabela 2.

Tabela 2. Níveis selecionados para os parâmetros de entrada.

\begin{tabular}{|c|c|c|c|c|c|c|c|}
\hline \multirow{2}{*}{ Parâmetros } & \multirow{2}{*}{ Unidade } & \multirow{2}{*}{ Notação } & \multicolumn{5}{|c|}{ Níveis } \\
\hline & & & -2 & -1 & 0 & 1 & 2 \\
\hline T.preaq & Ciclos & T.preaq & 2 & 5 & 8 & 11 & 14 \\
\hline I.preaq & $\%(k A)$ & I.preaq & 65 & 70 & 75 & 80 & 85 \\
\hline T.solda & Ciclos & T.solda & 8 & 10 & 12 & 14 & 16 \\
\hline I.solda & $\%(\mathrm{kA})$ & I.solda & 81 & 84 & 87 & 90 & 93 \\
\hline
\end{tabular}

Nota: $100 \%$ da corrente de soldagem representa $6 \mathrm{kA}$. Cada ciclo equivale a 0,01666 segundos.

Após a soldagem dos corpos de prova, a indentação foi medida por um relógio apalpador analógico com resolução de 0,01 mm juntamente com um traçador de altura, para fazer o ajuste fino durante a medição e um bloco magnético (ambos da marca Mitutoyo) com intuito de fixar as chapas de forma retilínea (Figura 2).

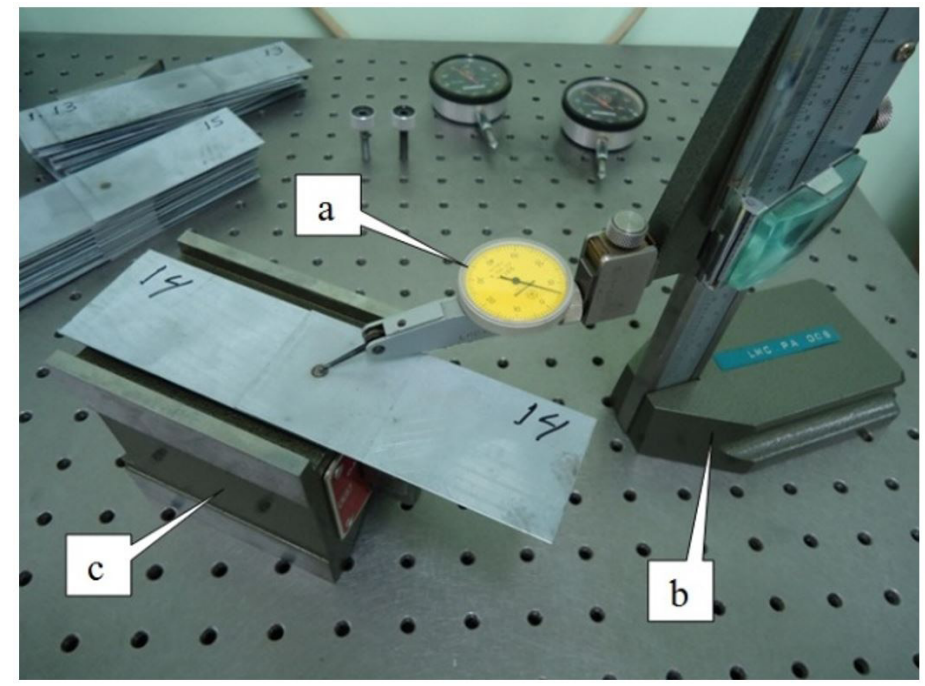

Figura 2. Medição da indentação da solda. (a) Relógio apalpador; (b) Traçador de altura; (c) Bloco magnético. Fonte: Baldim [8].

No ponto mais profundo da indentação (Figura 3) é referenciado o relógio apalpador. Em seguida, com intuito de encontrar os valores máximos e mínimos circula-se toda a superfície do ponto. Por último é feito a média dos valores encontrados. Este procedimento foi aplicado para minimizar o erro causado pela pequena deformação na superfície das chapas soldadas. Desta forma, o sinal do sensor de deslocamento do eletrodo foi formatado em um gráfico melhorando sua identificação conforme descrito na Figura 4. Assim, a indentação registrada pelo sensor de deslocamento do eletrodo (denominada IS) durante a soldagem, caracterizado pela diferença em módulo entre os pontos " $\mathrm{B}$ " e " $\mathrm{A}$ ", foi analisada com intuito comparativo à indentação medida pelo relógio apalpador (denominada IM). Esta comparação torna-se importante, na avaliação da confiabilidade do sinal de deslocamento do eletrodo para a determinação da indentação do ponto soldado, principalmente quando seu excesso ou sua falta possa refletir na qualidade da soldagem $[9,17]$. 


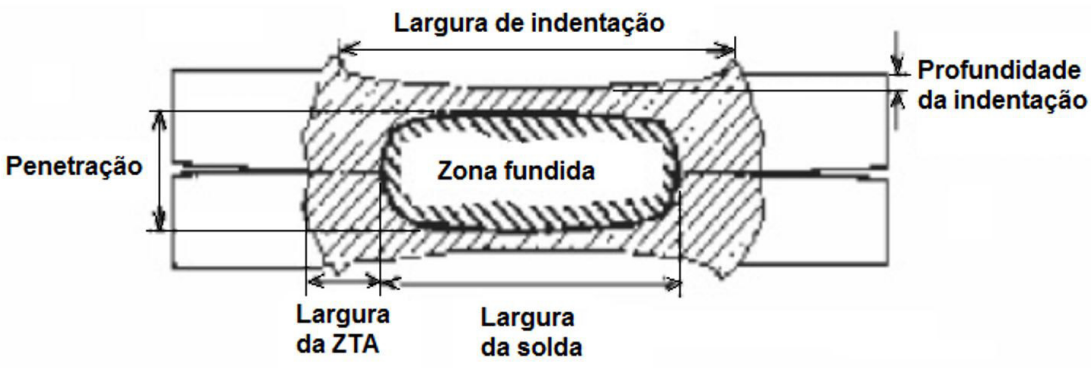

Figura 3. Geometria do ponto de solda. Adaptado de: Zhou et al. [7].

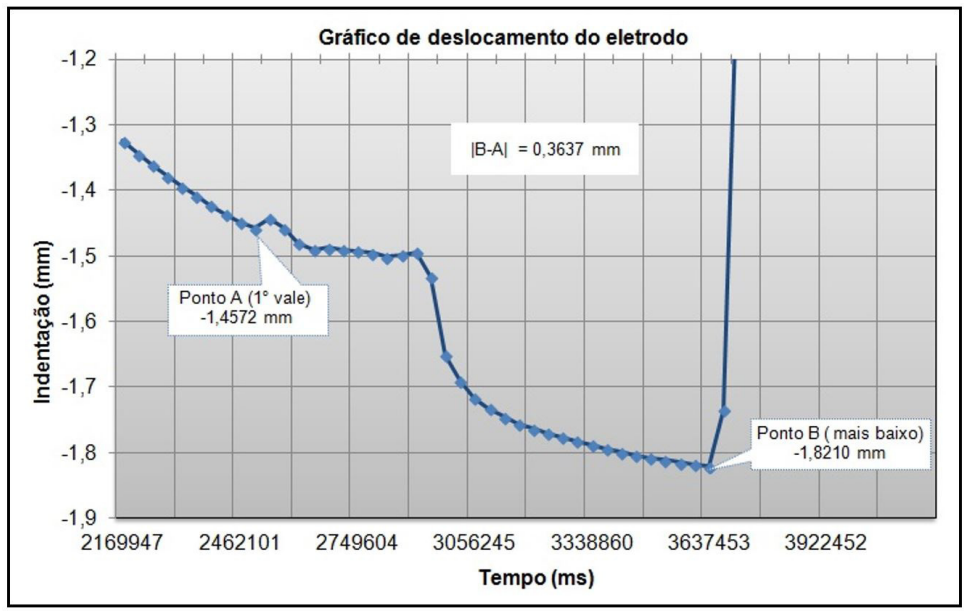

Figura 4. Gráfico de deslocamento do eletrodo. Fonte: Baldim [8].

\section{Resultados e Discussões}

Sabe-se que, os parâmetros RSW mais influentes para este experimento conforme descrito por Baldim [8] são, o tempo e a corrente de preaquecimento e soldagem. Tais parâmetros estão representados na Tabela 2 e Tabela 3 como T.preaq, I.preaq, T.solda e I.solda.

Durante a pesquisa, realizou-se um planejamento de experimentos DOE, com o intuito de conhecer a influência dos parâmetros de soldagem acima citados sobre as respostas de indentação, para tanto utilizou-se o relógio apalpador (IM) e o sinal de deslocamento do eletrodo (IS). No experimento foi empregado um arranjo experimental CCD (Central Composite Design) para explorar o maior espaço amostral possível.

Tabela 3. Coeficientes e suas interações no modelo reduzido.

\begin{tabular}{cccccc}
\hline \multirow{2}{*}{ Termos } & \multicolumn{2}{c}{ Coeficientes da IS } & & \multicolumn{2}{c}{ Coeficientes da IM } \\
\cline { 2 - 3 } \cline { 5 - 6 } & Coef & P-value & & Coef & P-value \\
Constant & 0,27470 & 0,000 & & 0,33240 & 0,000 \\
T.preaq & 0,03697 & $\mathbf{0 , 0 0 0}$ & & 0,05896 & $\mathbf{0 , 0 0 0}$ \\
I.preaq & 0,02205 & $\mathbf{0 , 0 0 1}$ & & 0,04479 & $\mathbf{0 , 0 0 1}$ \\
T.solda & 0,03629 & $\mathbf{0 , 0 0 0}$ & & 0,05812 & $\mathbf{0 , 0 0 0}$ \\
I.solda & 0,02470 & $\mathbf{0 , 0 0 0}$ & & 0,03438 & $\mathbf{0 , 0 0 7}$ \\
I.solda*I.solda & $-0,01193$ & $\mathbf{0 , 0 2 5}$ & & $-0,0227$ & $\mathbf{0 , 0 3 9}$ \\
T.preaq*I.preaq & 0,01547 & $\mathbf{0 , 0 3 1}$ & & 0,02531 & 0,084 \\
S & 0,0268 & & & 0,0559 & \\
$R^{2}$ (adj) & $82,18 \%$ & & & $73,01 \%$ & \\
\hline
\end{tabular}


A Tabela 3, indica os ajustes dos modelos em cada resposta analisada representados pelo $R^{2}(\operatorname{adj})$ demonstrando o grau de representatividade do modelo matemático de IS e IM em relação aos dados reais. Na mesma tabela, o desvio padrão é representado por $\mathrm{S}$ e os coeficientes da equação matemática por Coef. Quanto maior o Coef, maior a probabilidade da significância dos termos indicados nos valores de P-value. Assim, os valores de $P$-value $<0,05$ indicam os termos do arranjo experimental denominados significativos por influenciarem diretamente nas respostas de indentação IS e IM.

Observa-se na Tabela 3, destacados em negrito, os parâmetros do processo RSW de T.preaq, I.preaq, T.solda, I.solda e as interações I.solda*I.solda e T.preaq*I.preaq que apresentam significância para as respostas de IS e IM. Pode-se auferir que, tais parâmetros influenciam diretamente na indentação do ponto soldado. Por outro lado, nem todas as interações entre os parâmetros apresentaram valores significativos. Fato este, pode ser observado no valor de P-value $>0,05$ de T.preaq*I.preaq para a resposta IM.

Para avaliar a correlação existente entre a indentação medida pelo sensor IS e a medida pelo relógio apalpador IM, uma análise realizada por meio do software $\mathrm{MINTAB}^{\circledR}$ confirmou a existência de forte correlação entre ambas, registrando 0,941 para Pearson Correlation e 0,000 para P-Value. Assim, foi avaliado a diferença em módulo entre o valor final e o valor inicial de profundidade nos pontos A e B, ilustrados anteriormente na Figura 4, para cada corpo de prova. O resultado desta diferença indica a penetração do eletrodo no metal base. Neste contexto, questões relativas à confiabilidade desta diferença também podem ser sanadas pela avaliação comparativa entre os valores de IS e IM representados na Figura 5.

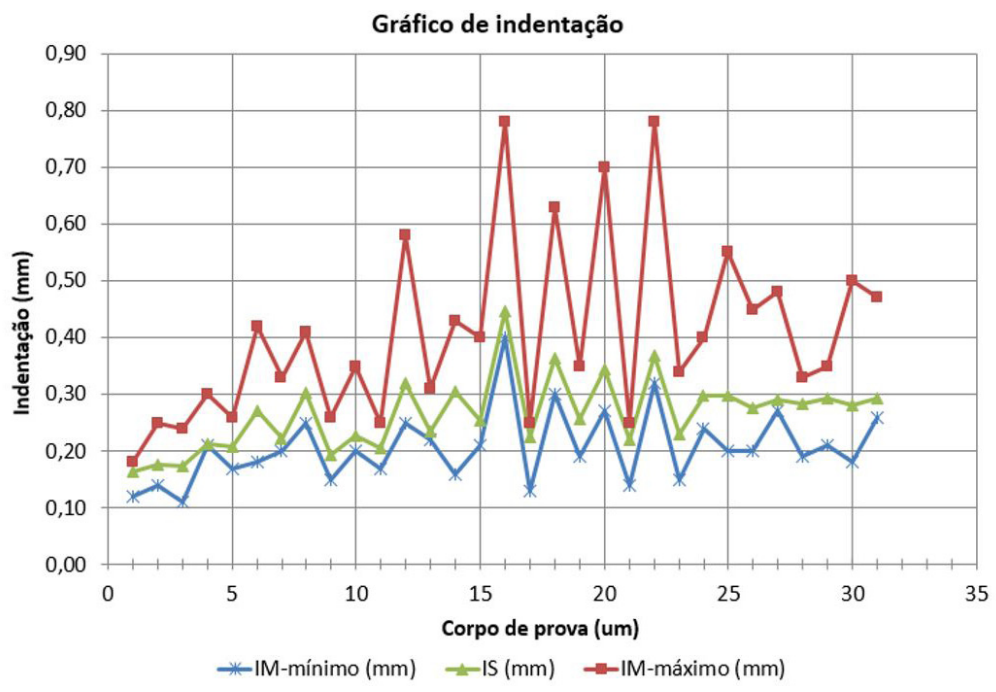

Figura 5. Gráfico comparativo entre IS e os limites de IM.

Observa-se na Figura 5 que, a linha representada pela cor verde ilustra a indentação IS que está compreendida entre o valor mínimo de indentação IM, representado pela linha na cor azul e o valor máximo de indentação IM representado pela linha na cor vermelha. Analisando os grandes picos nos valores máximos de IM pode-se dizer que, esta grande variação na amplitude esteja relacionada à variação na medição com o relógio apalpador. Este fato pode ser observado ao analisar o nível de $\mathrm{R}^{2}(\operatorname{adj})$ e o aumento do desvio padrão $\mathrm{S}$ nos valores de IM em comparação aos valores de IS, determinados anteriormente na Tabela 3. Para minimizar esta diferença medida pelo relógio apalpador um novo gráfico foi construído, desta vez considerando os valores médios de IM em comparação com IS mostrados na Figura 6.

Na Figura 6, pode-se observar que a diferença entre os valores de IS e IM estão mais próximos. Para este caso específico, um estudo estatístico R\&R (repetitividade e reprodutibilidade), que tem por objetivo validar o método de medição de IM, pode trazer benefícios ao aumentar o nível de $\mathrm{R}^{2}$ (adj) para esta resposta. Ainda neste 
Metodologia Não Destrutiva de Determinação da Indentação no Processo de Soldagem a Ponto por Resistência Elétrica em Chapas de Aços Galvanizados

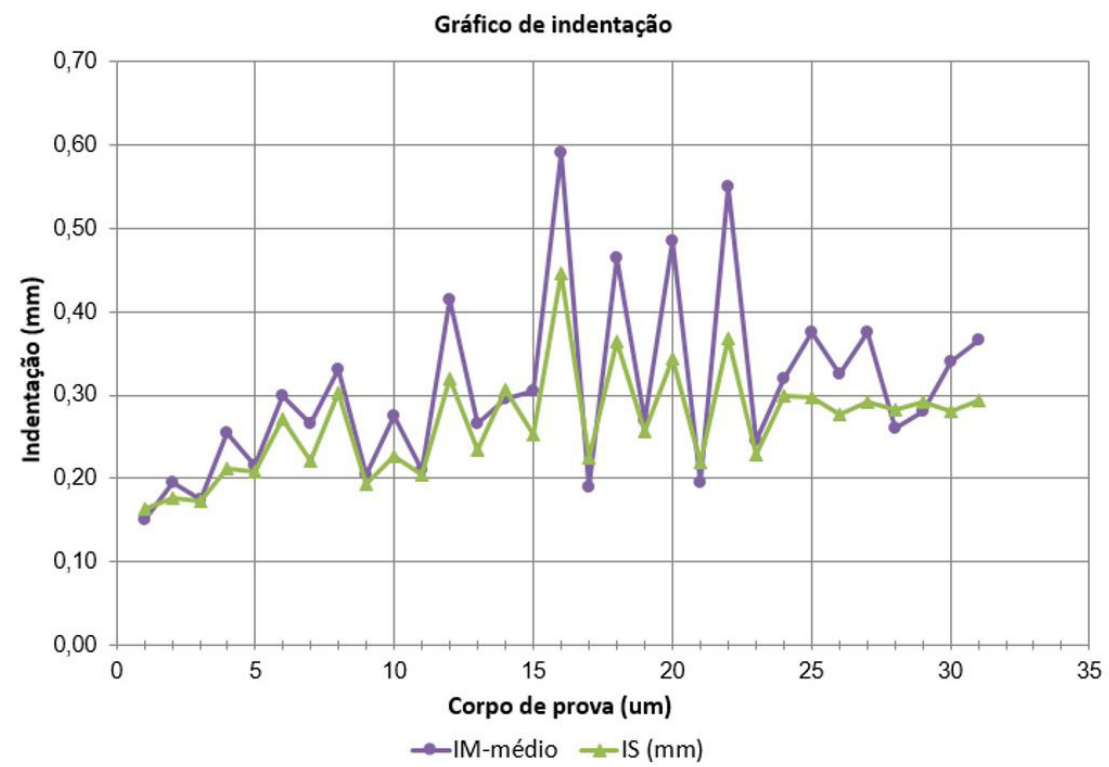

Figura 6. Gráfico comparativo entre IS e os valores médios de IM.

contexto, visando melhor ilustrar esta correlação entre os valores de IS e IM, novamente por meio do software MINTAB ${ }^{\circledR}$ foi construído o gráfico Fitted Line Plot, ilustrado na Figura 7, em que o modelo matemático de interação entre as indentações demonstrado pela Equação 1, consegue representar $88,2 \%$ da realidade.

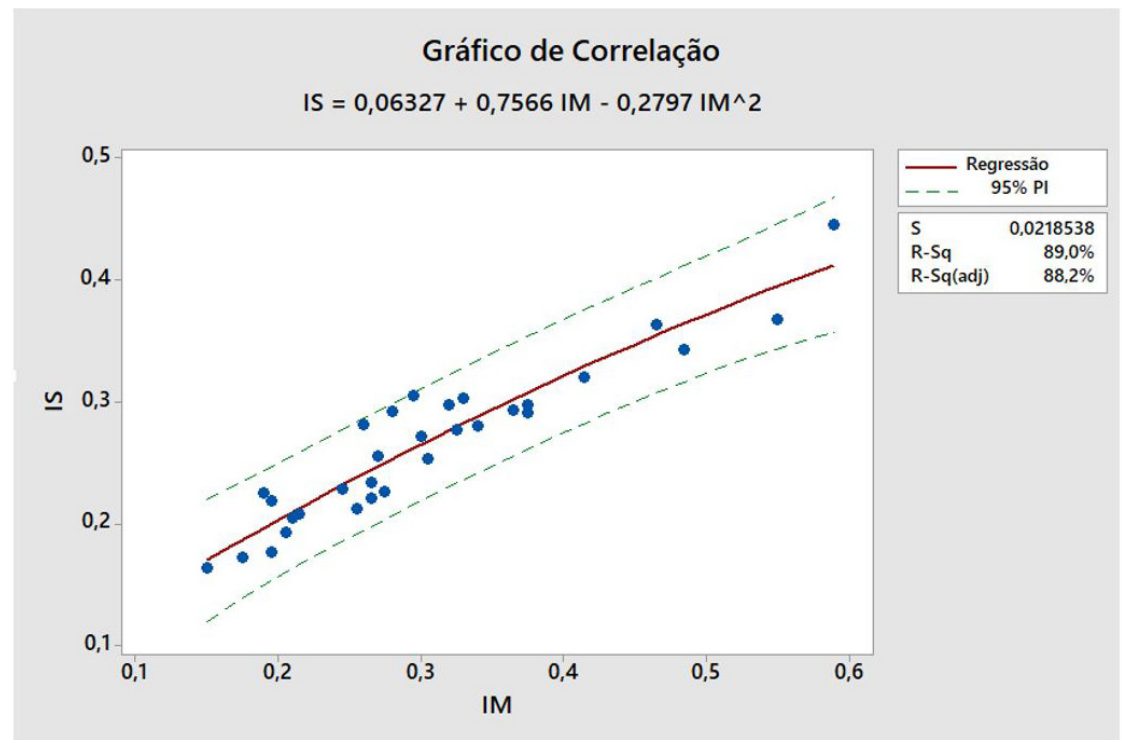

Figura 7. Gráfico de correlação entre os valores de IS e valores médios de IM.

$$
I S=0,06327+0,7566 I M-0,2797 I M^{2}
$$

Este fato pode ser muito interessante para o meio industrial, em que ocorre uma busca intensa pela redução de tempos de produção. Como resultado do experimento, tem-se a possibilidade de medir a indentação do ponto de solda por meio do sinal de deslocamento do eletrodo de forma on-line. Todavia, esta observação abre caminho para novas pesquisas no que diz respeito à utilização deste sinal de deslocamento do eletrodo, que tem como intuito caracterizar a qualidade da soldagem por resistência elétrica de forma não destrutiva durante o processo [16]. 


\section{Conclusões}

Baseado nos resultados obtidos neste trabalho, algumas conclusões podem ser feitas sobre esta metodologia:

> O baixo índice de $\mathrm{R}^{2}$ (adj) atrelado a grandes amplitudes entre o limite máximo e mínimo de IM pode estar relacionado à variação na medição pelo relógio apalpador. Fato este que, justifica a aplicação de um estudo de $R \& R$ (repetitividade e reprodutibilidade) para validação do método de medição de leitura pelo relógio apalpador.

- A influência dos parâmetros de tempo e corrente de soldagem e de preaquecimento, juntamente com a comprovação da existência da correlação ente IS e IM revelam que, o sinal de deslocamento do eletrodo IS pode ser tomado como parâmetro de avaliação da indentação do ponto de soldagem de forma on-line e não destrutiva.

- A forte correlação entre IM e IS abre caminhos para um vasto campo de pesquisa no aprimoramento e aumento da robustez da técnica de avaliação da indentação utilizando sensor de deslocamento do eletrodo.

\section{Agradecimentos}

Os autores gostariam de agradecer a Unifei pela infraestrutura laboratorial e pela Capes, pelo fornecimento de incentivos financeiros à pesquisa.

\section{Referências}

[1] Marques PV, Modenesi PJ, Bracarense AQ. Soldagem fundamentos e tecnologia. Belo Horizonte: Ed. UFMG, 2009. p. 293-306.

[2] Pereira MA, Ferreira JM, Loureiro A, Costa JDM, Bártolo PJ. Effect of process parameters on the strength of resistance spot welds in 6082-T6 aluminium alloy. Materials \& Design. 2009;31(5):24542463. https://doi.org/10.1016/j.matdes.2009.11.052.

[3] Vargas JE. Estudo da formação, geometria e resistência do ponto na soldagem por resistência [dissertação de mestrado]. Brasília: Universidade de Brasília; 2006.

[4] Azambuja S, Barcelos EJ. Soldagem de chapas zincadas convencionais pelo processo a ponto por resistência elétrica. In: Associação Brasileira de Soldagem. Anais do $18^{\circ}$ Encontro Nacional sobre Tecnologia de Soldagem; 1987; Rio de Janeiro, Brasil. São Paulo: ABS; 1987. p. 1-18.

[5] Usinas Siderurgicas de Minas Gerais S.A - Usiminas. Material de treinamento introdutório aos conhecimentos básicos do processo de soldagem por resistência. Pouso Alegre: Automotiva Usiminas; 2010. $34 \mathrm{p}$.

[6] Alenius M, Al E. Exploring the mechanical properties of spot welded dissimilar joints for stainless and galvanized steels. Welding Journal. 2006;85(12):305s-313s.

[7] Zhou M, Zhang H, Hu SJ. Relationships between quality and attributes of spot welds. Welding Journal. 2003;(Suppl):72s-77s.

[8] Baldim AC. Análise do efeito da eliminação da camada de zinco na qualidade da solda a ponto em chapas de aços galvanizados [dissertação de mestrado]. Itajubá: Universidade Federal de Itajubá; 2014.

[9] Medar Comércio e Serviços Ltda. Treinamento básico do processo de soldagem por resistência para operadores e preparadores de máquina. Pouso Alegre: Usiminas; 2005. 81 p.

[10] Tecnowelding Comércio Ltda. Fundamentos do processo de soldagem a resistência. Curitiba: Tecnowelding Comércio Ltda; 2004. 70 p.

[11] Lee SR, Choo YJ. A quality assurance technique for resistance spot welding using a neuro-fuzzy algorithm. Journal of Manufacturing Systems. 2001;20(5):320-328. http://dx.doi. org/10.1016/S0278-6125(01)80051-0.
[12] Aslanlar S. The effect of nucleus size on mechanical properties in electrical resistance spot welding of sheets used in automotive industry. Materials \& Design. 2006;25(2):125-131. http://dx.doi. org/10.1016/j.matdes.2004.09.025.

[13] American Welding Society-AWS. AWS/SAE D8.9M: Recommended Practices for Test Methods for Evaluating the Resistance Spot Welding Behavior of Automotive Sheet Steel Materials. Florida: American Welding Society; 2002.

[14] Wolff MA, Silva RF, Vilarinho LO. Determinação dos campos de soldabilidade para o aço dual-phase 600 em equipamentos de soldagem a ponto AC e MFDC. Soldagem \& Inspeção. 2010;15(3):200208. http://dx.doi.org/10.1590/S0104-92242010000300006.

[15] Stocco D, Gonçalves R. Caracterização dos defeitos encontrados em solda a ponto utilizando a técnica do ultrassom, apoiado à exames metalográficos. In: Associação Brasileira de Ensaios Não Destrutivos. Proceedings of the 3rd Pan-American Conference for Nondestrutive Testing; 2003 Jun 2-6; Rio de Janeiro, Brasil. São Paulo: ABENDE; 2003. 9 p, 2003.

[16] Xinmin L, Xiaoyun Z, Yansong Z, Guanlong C. Weld quality inspection based on online measured indentation from servo encoder in resistance spot welding. IEEE Transactions on Instrumentation and Measurement. 2007;56(4):1501-1505. http://dx.doi.org/10.1109/TIM.2007.900423.

[17] Gedeon SA, Eagar TW. Resistance spot welding of galvanized steel, part II: mechanisms of spot weld nugget formation. Metallurgical Transactions. B, Process Metallurgy. 1986;17b(4):887-901. http://dx.doi.org/10.1007/BF02657152.

[18] Montgomery DC. Design and analysis of experiments. New York: John Wiley; 2005. 643 p.

[19] Myers RH, Montgomery DC, Anderson-Cook CM. Response surface methodology. 3rd ed. New York: John Wiley \& Sons; 2009.

[20] Santos FBS. Estudo da eficiência do processo de soldagem de pontos por resistência elétrica utilizada na indústria automotiva [dissertação de mestrado]. Rio de Janeiro: Universidade Federal Fluminense; 2006. 